\title{
Potential of exocellular carbohydrate antigens of Staphylococcus epidermidis in the serodiagnosis of orthopaedic prosthetic infection
}

\author{
P. A. LAMBERT, A. VAN MAURIK, S. PARVATHAM, Z. AKHTAR, A. P. FRAISE* and S. J. KRIKLER $\dagger$ \\ Microbiology Research Group, Pharmaceutical Sciences Institute, Aston University, Birmingham B4 7ET, \\ *Hospital Infection Research Laboratory, City Hospital NHS Trust, Dudley Road, Birmingham B18 $70 H$ and \\ †Royal Orthopaedic Hospital, Birmingham B31 2AP
}

\begin{abstract}
The potential of exocellular carbohydrate antigens of Staphylococcus epidermidis as markers of infection in bone was investigated by immunoblotting and enzyme-linked immunosorbent assay (ELISA). Exocellular antigens were prepared by gel filtration chromatography of concentrated brain heart infusion culture supernates. The antigenic material appeared as diffuse bands between 24 and $32 \mathrm{kDa}$ on the immunoblots and was not susceptible to digestion with trypsin, indicating that the response in the patients was to non-protein (polysaccharide or teichoic acid, or both) exocellular material. Significant differences were detected between the immunoblot antigen profiles for serum IgG from patients with $S$. epidermidis bone infection and those with an uninfected prosthetic joint. Thirteen of 16 patients with $S$. epidermidis prosthetic joint infection showed an elevated serum IgG level by ELISA compared with controls with uninfected joints. However, the antigen was not specific for $S$. epidermidis bone infection; high levels of IgG were also detected in patients with other serious staphylococcal and streptococcal infections. The ELISA test may be valuable in distinguishing between staphylococcal infection of joints and aseptic loosening by excluding cases of infection.
\end{abstract}

\section{Introduction}

Staphylococcus aureus and coagulase-negative staphylococci (CNS) are the most common causes of prosthetic joint infection [1]. It is important to distinguish between septic and aseptic loosening of a prosthesis, as treatment of the two conditions differs [2]. Early accurate diagnosis is a major clinical problem in prosthetic infection and serodiagnostic methods may offer rapid, non-invasive identification of the infecting organism, allowing prompt intervention with appropriate antimicrobial therapy. The immune response to exocellular proteins of $S$. aureus in serum from patients with osteomyelitis has been investigated in an earlier study and a serodiagnostic test was devised $[3,4]$. The serum IgG response towards these antigens in patients with infection associated with artificial joints was significantly higher than in patients with uninfected joints or in healthy normal

Received 5 June 1995; revised version accepted 27 Sept. 1995.

Corresponding author: Dr P.A. Lambert.

$\nmid$ Present address: Dr S.J. Krikler, Coventry and Warwickshire Hospital, Coventry CV4 1FH. controls $[3,4]$. The choice of exocellular proteins was based on previous Western blotting studies of patient response to cellular and exocellular antigens of S. aureus $[5,6]$.

S. epidermidis accounts for $50-80 \%$ of CNS isolates from implanted medical device infections [7]. Its ability to adhere to foreign bodies and to produce extracellular slime during the course of colonisation is thought to be important in the development of these infections [8]. There is considerable debate as to the chemical nature of extracellular slime produced by $S$. epidermidis. Different polysaccharides rich in mannose [9], galactose [10], glucose [11] or hexosamine [12] have been identified and described as extracellular slime substance, capsular polysaccharide adhesin, slime associated antigen and intercellular adhesin, respectively. By contrast, most of the extracellular polymeric material produced in a chemically defined medium has been identified as a glycerol phosphate teichoic acid [13]. In comparison with $S$. aureus, $S$. epidermidis produces a limited number of exocellular enzymes. Lipase, esterase, DNAase and $\alpha$ - and $\beta$ haemolysins have been detected with a mouse model of foreign body infection [14] and a urease has been 
linked with the degradation of polyurethane and colonisation of catheters [15]. The present study investigated the potential of exocellular $S$. epidermidis antigens in the serodiagnosis of orthopaedic infections.

\section{Materials and methods}

\section{Strains}

Bacterial strains were isolated from orthopaedic prosthetic infections at the Royal Orthopaedic Hospital, Birmingham and identified as $S$. epidermidis by the API-Staph identification system (API Laboratory Products Ltd, Basingstoke).

\section{Growth and preparation of antigen fractions by fast protein liquid chromatography (FPLC)}

Cultures were grown in $100 \mathrm{ml}$ of Brain Heart Infusion Broth (BHI, Difco) for $18 \mathrm{~h}$ at $37^{\circ} \mathrm{C}$ with vigorous shaking in a rotary incubator. Cells were harvested by centrifugation $(10000 \mathrm{~g}, 10 \mathrm{~min})$ and the culture supernates were retained for immunoblotting studies. For purification of the antigens, the culture supernate $(100 \mathrm{ml})$ was first concentrated 20 -fold by freeze drying and reconstituting in $5 \mathrm{ml}$ of water. After centrifugation at $15000 \mathrm{~g}$ for $5 \mathrm{~min}$ to remove any residual insoluble material, the concentrated supernate (in 0.5-ml volumes) was applied to a Superose 12 10/30 gel filtration column (Pharmacia) equilibrated with water. The column was washed with water at $0.4 \mathrm{ml} / \mathrm{min}$; the eluate was monitored at $280 \mathrm{~nm}$ and 0.8-ml fractions were collected (Pharmacia FPLC equipment). The void volume of the column (determined by elution of blue dextran) was $8 \mathrm{ml}$. The high mol.wt proteins $(>65 \mathrm{kDa})$ were eluted from the column in fractions 10 and 11 . Fractions 12-19 containing exocellular polysaccharide or teichoic acid, or both, were pooled and used in the immunoblotting and ELISA studies. Low mol.wt exocellular bacterial products and residual components of the BHI growth medium were eluted in fractions 20-38. Therefore, most of the exocellular proteins, all of the low mol.wt bacterial products and culture medium components were excluded from the immunoassays.

\section{Trypsin digestion}

A $1.5-\mathrm{ml}$ portion of the pooled fractions $12-19$ from the Superose 12 column (above) was treated with $0.1 \mathrm{ml}$ of $0.5 \mathrm{M}$ ammonium carbonate buffer ( $\mathrm{pH} 7.6)$ containing $1 \mathrm{mg}$ of bovine pancreatic trypsin (Sigma) for $18 \mathrm{~h}$ at $20^{\circ} \mathrm{C}$. The digested material was subjected to SDS-PAGE and immunoblotting as described below.

\section{Sera}

Serum was collected by venepuncture from three categories of subject: healthy adults (5); patients with prosthetic joints, but no known infection, who were being admitted for surgery (29); and patients with prosthetic joints infected by $S$. epidermidis (16). Further sera were from patients with prosthetic joints infected by $S$. aureus (5) as reported previously [3], patients with $S$. epidermidis infection of intra venous catheters (5) and single serum samples from patients with $S$. aureus infective endocarditis, group A streptococcal infective endocarditis and Enterococcus faecalis osteomyelitis. All sera were stored at $-20^{\circ} \mathrm{C}$.

\section{SDS-PAGE and immunoblotting}

Proteins were separated by SDS-PAGE with the Lugtenberg gel system [16]. Culture supernates were diluted with an equal volume of denaturing buffer and heated at $100^{\circ} \mathrm{C}$ for $10 \mathrm{~min}$. Ten- $\mu 1$ samples were applied to the wells of polyacrylamide $12 \% \mathrm{w} / \mathrm{v}$ gels, electrophoresed at $200 \mathrm{~V}$ (BioRad Mini Protean II apparatus) and stained with Coomassie Blue. For immunoblotting, a sample of denatured material was applied to a single $10 \mathrm{~cm}$ wide track on the gel. Immediately after electrophoresis the pattern of separated bands was transferred from the gel to a nitrocellulose sheet $(0.45 \mu \mathrm{m}$ pore size, BioRad $)$ by electrophoresis $\left(100 \mathrm{~V} ; 1 \mathrm{~h} ; 5^{\circ} \mathrm{C}\right)$ in Tris-glycine buffer (25 mM Tris, $192 \mathrm{mM}$ glycine, $\mathrm{pH} 8.3$ ) containing methanol $20 \% \mathrm{v} / \mathrm{v}$ by the BioRad Mini Trans Blot system. Unbound sites on the nitrocellulose sheets were blocked with TBS-Tween $(0.01 \mathrm{M}$ Tris- $\mathrm{HCl}, \mathrm{pH} 7.4$, $\mathrm{NaCl} 0.9 \% \mathrm{w} / \mathrm{v}$, Tween-20 $0.3 \% \mathrm{v} / \mathrm{v}$ ). Sheets were cut vertically into $1-\mathrm{cm}$ wide strips and the strips were immersed in patient serum diluted 1 in 200 in TBSTween for $3 \mathrm{~h}$ at $25^{\circ} \mathrm{C}$. After washing the strips three times in TBS-Tween, bound IgG was detected by its reaction with protein A-horseradish peroxidase conjugate $(50 \mu \mathrm{g} / \mathrm{ml}$ in TBS-Tween, Sigma) for $2 \mathrm{~h}$ at $25^{\circ} \mathrm{C}$. Strips were washed three times in TBS and bands were visualised by addition of a solution containing 4chloronaphthol $25 \mu \mathrm{g} / \mathrm{ml}$ in $0.01 \mathrm{M}$ Tris- $\mathrm{HCl}(\mathrm{pH} 7.4)$ and $10 \mu \mathrm{l}$ of $\mathrm{H}_{2} \mathrm{O}_{2} 20 \% \mathrm{v} / \mathrm{v}$.

\section{ELISA}

Microtitration plates (Immulon 2, Dynatech) were coated with either crude culture supernate or pooled fractions from the FPLC purification as described previously [4]. Antigen preparations were diluted with 100 volumes of sodium carbonate/bicarbonate buffer $(0.05 \mathrm{M}, \mathrm{pH} 9.6)$ before coating wells with $100 \mu \mathrm{l}$ at $4^{\circ} \mathrm{C}$ for $18 \mathrm{~h}$. After removal of excess antigen wells were washed with TBS-Tween. Unbound sites were blocked by incubation in the same buffer $\left(1 \mathrm{~h}, 4^{\circ} \mathrm{C}\right)$. Patient sera $(100 \mu \mathrm{l})$, diluted 1 in 800 in TBS-Tween, unless indicated otherwise, were added to the wells and incubated for $2 \mathrm{~h}$ at $4^{\circ} \mathrm{C}$. After removal of excess serum and washing with TBS-Tween, bound IgG was detected by addition of $100 \mu \mathrm{l}$ of protein A-horseradish peroxidase conjugate $(0.5 \mu \mathrm{g} / \mathrm{ml}$ in TBS-Tween, Sigma) and incubation for $2 \mathrm{~h}$ at $4^{\circ} \mathrm{C}$. After removal of conjugate and washing with TBS-Tween, $100 \mu \mathrm{l}$ of 
chromogenic substrate were added to each well. The substrate contained $10 \mathrm{mg}$ of 3,3',5,5'-tetramethylbenzidine (Sigma) dissolved in $1 \mathrm{ml}$ of dimethyl sulphoxide and diluted into $100 \mathrm{ml}$ of sodium acetate/citrate buffer $(0.1 \mathrm{M}, \mathrm{pH} 6.0)$ containing $10 \mu 1$ of $\mathrm{H}_{2} \mathrm{O}_{2} 20 \%$ $\mathrm{v} / \mathrm{v}$. When the colour had developed sufficiently $(5 \mathrm{~min}$ at $20^{\circ} \mathrm{C}$ ), the reaction was stopped by addition of $50 \mu \mathrm{l}$ of sulphuric acid $(1 \mathrm{M})$ to each well and the optical density was measured at $450 \mathrm{~nm}$ with an Anthos 2001 plate reader (Anthos Labtec Instruments). Assays were also performed with goat anti-human IgM-peroxidase or goat anti-human IgA-peroxidase conjugates (Sigma) in place of protein A-peroxidase to measure IgM and IgA levels in sera. Control ELISA assays with uncoated wells (blocked with Tween 20) or wells coated with material prepared from brain heart infusion medium alone (processed by FPLC in the same way as the culture supernate) and patient sera and conjugates produced no colour.

\section{Results}

\section{Immunoblotting studies}

Fig. 1 shows the protein profiles of culture supernates of six strains of $S$. epidermidis grown in BHI. The number of bands varied considerably between the strains; strain 5 produced the greatest number. Studies with other laboratory growth media (nutrient broth, tryptic soya broth, Mueller-Hinton broth and IsoSensitest broth) also showed a range of exocellular proteins produced by these strains (data not shown), with some variation in the number of bands and their relative intensities. Strain 5 grown in BHI was selected for further immunoblotting studies, as this combination produced the greatest number of protein bands and was considered to best represent the maximum range of exocellular proteins produced by $S$. epidermidis.
Fig. 2 shows the strip immunoblots of the crude culture supernate from strain 5 grown in BHI (i.e. the same material as Fig. 1, lane 5) probed with sera (diluted 1 in 200) from three patients with uninfected prosthetic joints, sera from three patients with $S$. epidermidis prosthetic joint infection and pooled serum from healthy donors. The most striking feature is the lack of major differences in the antigenic profiles between the different groups of patients and the healthy donor serum. A number of sharp bands appeared in all lanes, most notably at 65,80 and $106 \mathrm{kDa}$. These are assumed to be proteins present in the culture supernate because of their sharp resolution on SDS-PAGE and immunoblotting. Screening of the sera from all 16 patients with $S$. epidermidis bone infection and 29 non-infected patients with bone prostheses gave essentially the same results for the antigenic profiles as shown in Fig. 2, suggesting that the exocellular proteins are not reliable markers for serodiagnosis of bone infection.

There was also diffuse material migrating in the 24 $65-\mathrm{kDa}$ region of the blots which appeared to react more strongly with the sera from infected patients than with sera from uninfected patients or the healthy donor pooled serum. Because of the diffuse appearance of this material and its failure to stain with Coomassie Blue, it is assumed not to be protein, but of polysaccharide or teichoic acid composition. Attempts were made to purify this material with a gel filtration system based on that used by Mack et al. [12]. Fig. 3 shows the strip immunoblots of this purified antigenic material probed with the same sera as shown in Fig. 2. Most of the sharp protein bands were removed in the purification and the remaining polysaccharide/teichoic acid material concentrated so that there was now a clear distinction between the antigenic profiles of the infected (lanes 4-6) and non-

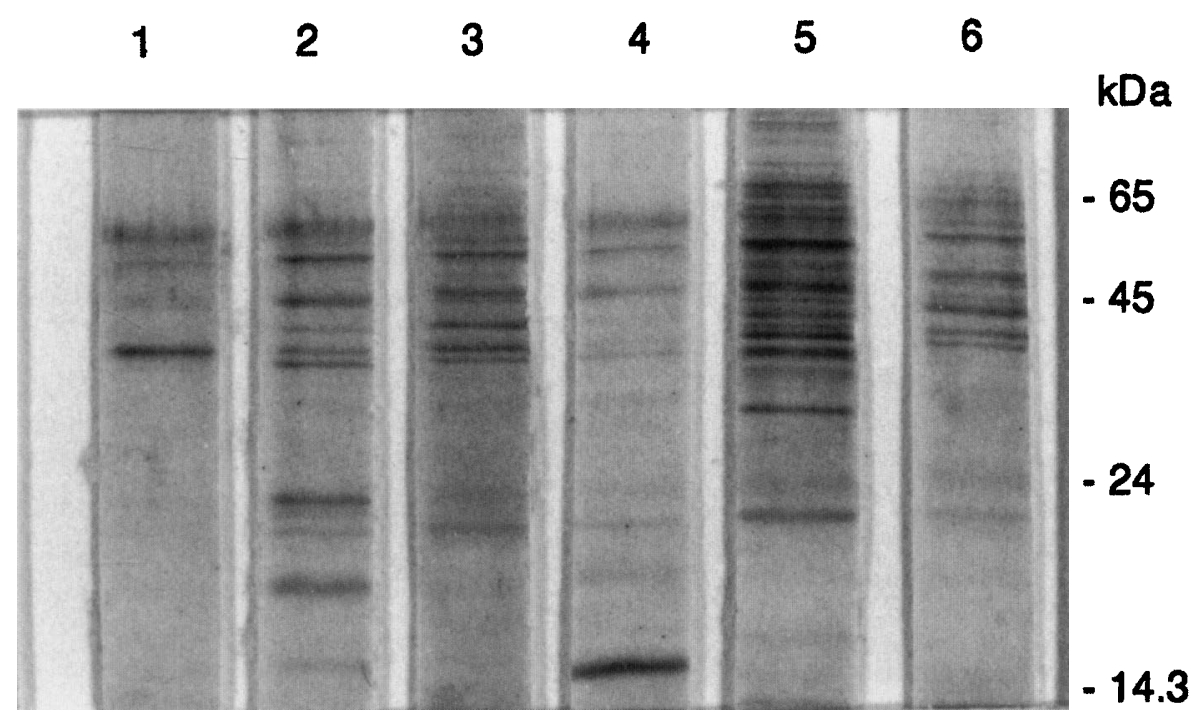

Fig. 1. Coomassie Blue-stained SDS-PAGE gel of BHI culture supernate of six strains of $S$. epidermidis bone infection isolates (lanes 1-6). Mol. wts determined from standard protein markers are shown. 


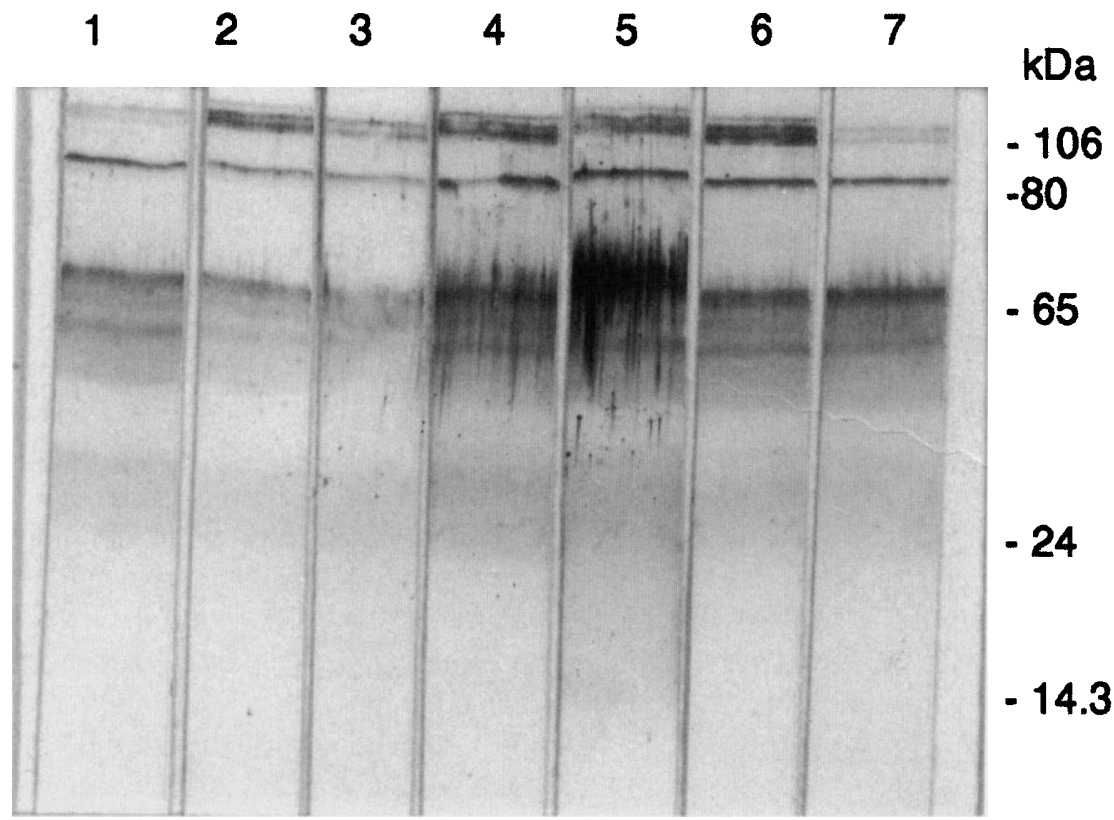

Fig. 2. Immunoblots of exocellular antigens in culture supernate of $S$. epidermidis (strain used in lane 5, Fig. 1) grown in BHI. Lanes 1-3, sera (diluted 1 in 200) from patients with uninfected prosthetic joints; 4-6, sera (diluted 1 in 200) from patients with prosthetic joint infection due to $S$. epidermidis; 7, pooled sera from 10 healthy donors.

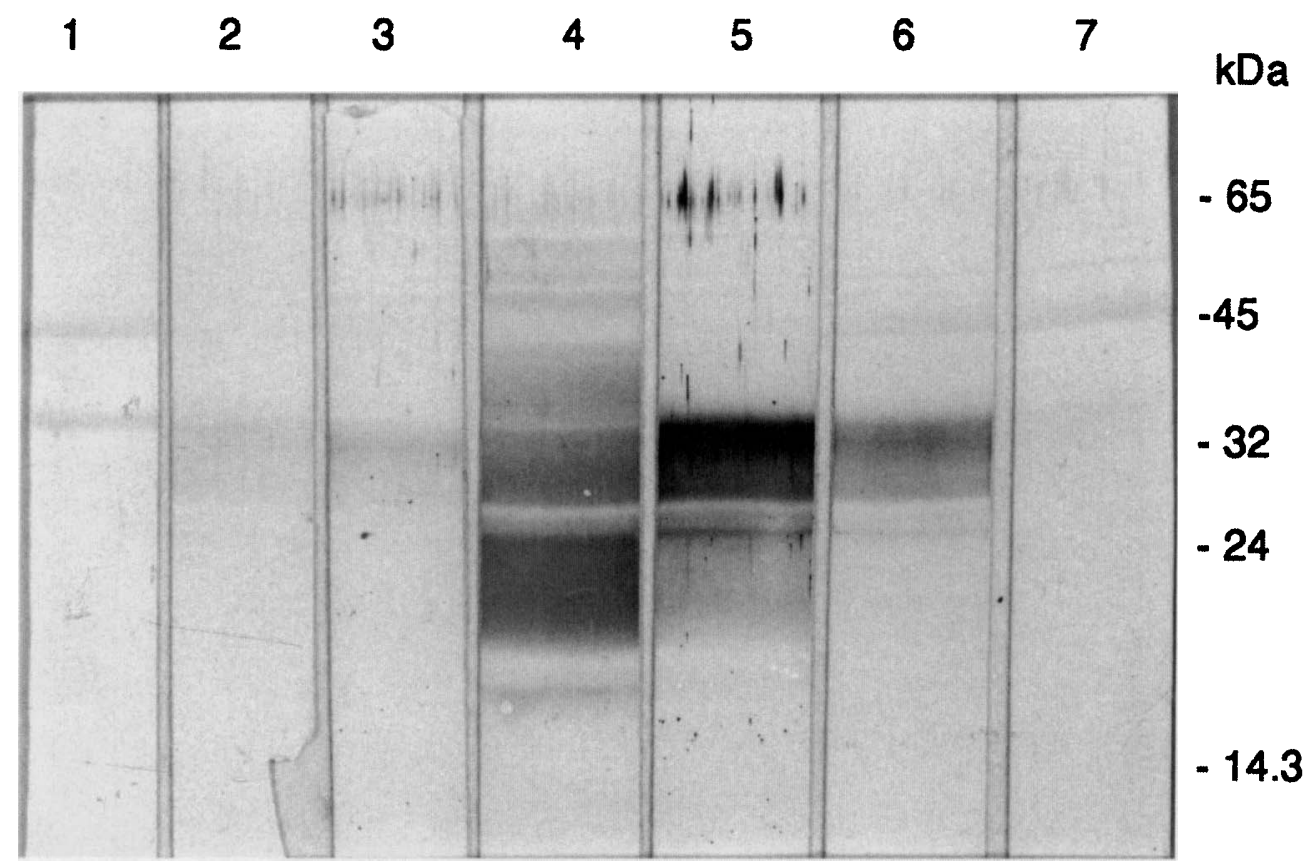

Fig. 3. Immunoblots prepared as for Fig. 2 with purified exocellular antigen instead of crude BHI culture supernate.

infected (lanes 1-3) patient sera. Similar results were obtained for sera from the other 13 infected patients; these all showed a strong response towards the diffusely migrating material between 25 and $32 \mathrm{kDa}$ on the blots. Blots with sera from over 100 noninfected patients with prosthetic joints showed no reaction with this material. Further evidence for the non-protein nature of the positively reacting antigenic material of the immunoblots was obtained by digestion with trypsin. Fig. 4 shows the antigenic profiles of this material before and after treatment with trypsin on immunoblots probed with serum from an infected patient (as in Fig. 3, lane 5). Although the strongly reacting material around $32 \mathrm{kDa}$ was reduced by trypsin treatment, overall the immunoblot reaction remained positive, suggesting that most of the antigenic material was not protein.

\section{ELISA studies}

To investigate the quantitative nature of the IgG response an ELISA test was developed with micro- 


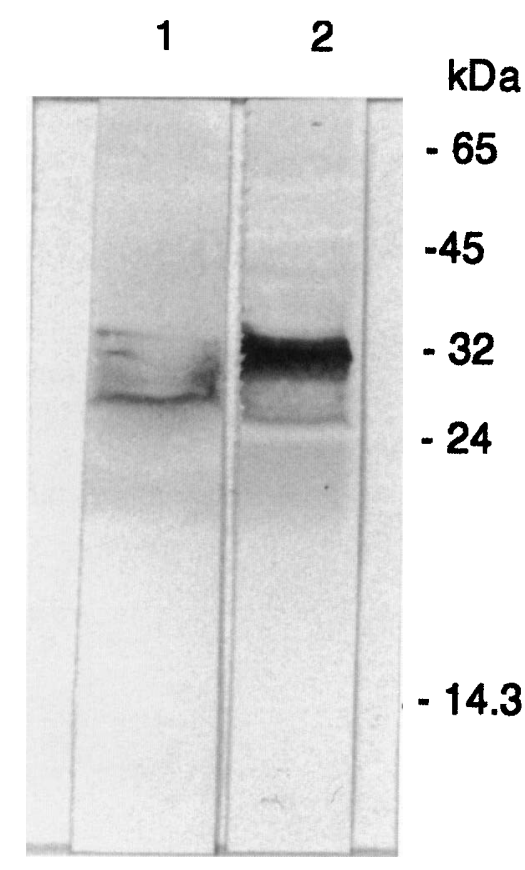

Fig. 4. Immunoblots of purified exocellular antigens before (lane 2) and after (lane 1) treatment with trypsin. The blots were probed with the serum used for lanes 5 of Figs 2 and 3.

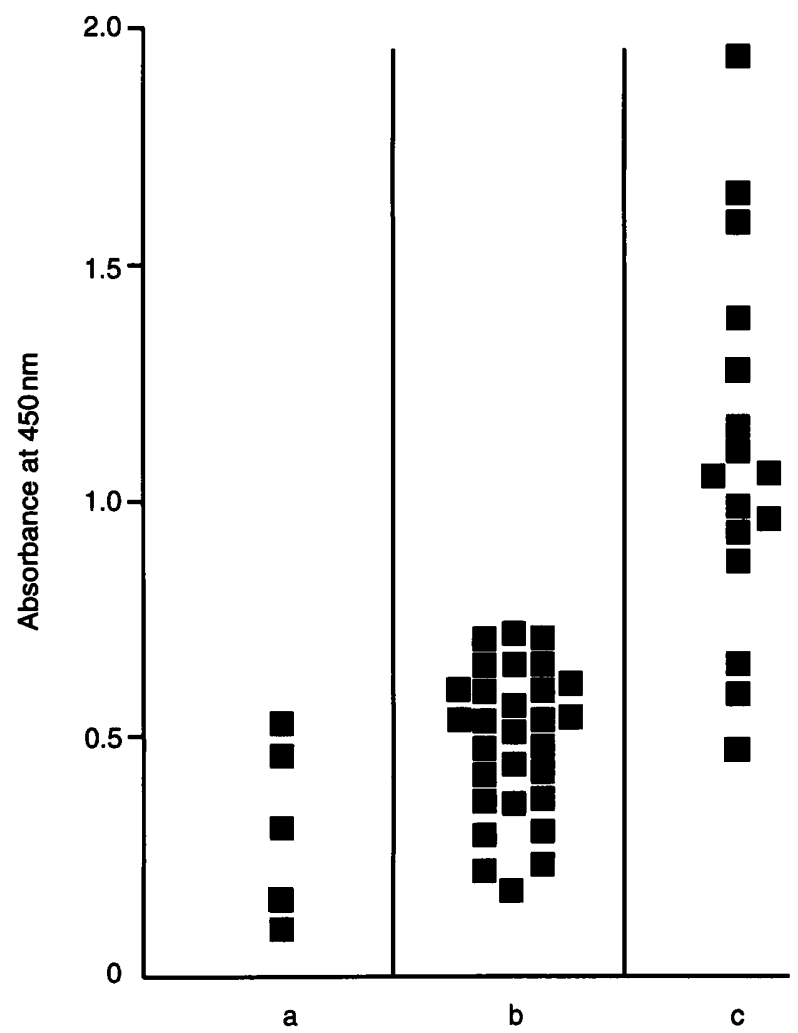

Fig. 5. ELISA absorbance $(450 \mathrm{~nm})$ values with sera (diluted 1 in 800 ) from: a, healthy adults, b, patients with an uninfected prosthetic joint and no other known infection c, patients with a prosthetic joint infected with $S$. epidermidis. titration plates coated with the polysaccharide/teichoic acid material. The test was extremely sensitive, colour production being detected with dilutions as high as 1 in 100000 with sera from some infected patients. Comparison of the ELISA titration curves for sera from infected and non-infected patients showed that the clearest distinction between the patient groups was at a serum dilution of 1 in 800 (Fig. 5). Mean absorbance values were significantly higher for sera from infected patients (1.112, SD 0.415 versus 0.573 , SD 0.136; $\mathrm{p}<0.0001$ ). An ELISA with unpurified culture supernate as the antigen showed no significant difference between sera from the two patient groups.

The specificity of the ELISA was investigated by examining sera from patients with other gram-positive bacterial infections. The mean absorbance of sera from five patients with $S$. aureus infection of prosthetic joints was not significantly different from the value obtained for the sera from patients with S. epidermidis joint infections (0.793, SD 0.477 versus 1.112 , SD $0.415 ; \mathrm{p} 0.164)$. Other sera that gave positive results in the ELISA test included: one from a patient with $S$. aureus endocarditis (absorbance 1.420); one from a patient with endocarditis due to a group A streptococcus (1.417), one from a patient with Enterococcus faecalis osteomyelitis (1.230) and three from patients with $S$. epidermidis infection of intravenous catheters $(0.890,0.729$ and 0.746$)$. No significant IgM or IgA levels were detected in any of the sera when the assay was run with goat anti-human IgM- or IgAperoxidase conjugates.

\section{Discussion}

Previous studies with sera from patients with prosthetic joint infection due to $S$. aureus have established that exocellular proteins are prominent antigens that can be exploited in the design of a serodiagnostic ELISA for $S$. aureus bone infection $[3,4]$. It was reasoned that the localised nature of the infection prevented immune detection of cellular antigens. By contrast, soluble products released from the organisms were readily detected by the immune system, resulting in elevated IgG levels compared with those of normal individuals or those with uninfected prosthetic joints. When applying the same approach to sera from patients with $S$. epidermidis infection of prosthetic joints it was found that exocellular proteins of this organism were of no serodiagnostic value for this bone infection. Extensive immunoblotting investigation with different strains, media and patient sera failed to reveal major differences in serum IgG levels towards proteins in crude culture supernates. However, elevated levels of IgG towards antigenic material purified from BHI culture medium by gel filtration were observed. The purification procedure removed most of the exocellular protein, leaving material presumed to be polysaccharide [12] or teichoic acid, or both [13] as the major 
exocellular antigens. The precise nature of this material has not yet been determined and full immunochemical characterisation is necessary to identify the components. Resistance to trypsin, failure to stain with Coomassie Blue and a diffuse pattern of migration on SDS-PAGE in the 24-32-kDa region (for protein markers) indicates that the material is anionic, low mol.wt and possibly lipid-containing. There are few reports of the behaviour of gram-positive wall, membrane and capsular components on SDS-PAGE. Lipocarbohydrate antigens of Clostridium difficile [17] and capsular polysaccharide of $S$. aureus [18] have been shown to produce ladder patterns on immunoblotting or staining with alcian blue and silver. The lipoteichoic acid and surface polysaccharides of $S$. epidermidis might behave in a similar manner. Several different exopolysaccharides thought to be important in adhesion to surfaces and in biofilm formation have been described in S. epidermidis [9-12]. The teichoic acid material described by Hussain et al. [13] and lipoteichoic acid [19] could also be involved in adhesion or biofilm formation. Their appearance in culture supernate suggests that turnover of the cell wall occurs during culture, releasing the same wall (e.g., teichoic acid and peptidoglycan) and membrane (e.g., lipoteichoic acid) components. This could also occur during infection of bone, resulting in detection by the patient's immune system. Further evidence that the antigen did not contain protein or peptidoglycan was obtained by studying the precipitins produced by the material with patient serum following immunodiffusion in agarose gels. The appearance of the precipitins was unaffected by pretreatment of the antigen with either trypsin or lysostaphin $\left(18 \mathrm{~h}\right.$ at $\left.37^{\circ} \mathrm{C}\right)$ or by heating at $100^{\circ} \mathrm{C}$ for $10 \mathrm{~min}$ before immunodiffusion (data not shown). We have also shown that a single absorption of patient sera with washed whole cells of $S$. epidermidis reduces the ELISA response to that of normal serum. This indicates that the antigens are present in the wall and exposed on the surface. Both properties are consistent with their identification as wall and membrane teichoic acids or surface-associated polysaccharides.

These initial studies suggest that a simple ELISA based on non-protein antigens may provide a convenient means of diagnosing $S$. epidermidis infection of prosthetic joints, or at least of excluding $S$. epidermidis infection where mechanical loosening is suspected. The ELISA might also be useful for monitoring of patients after insertion of artificial joints providing an early indication of $S$. epidermidis infection. However, there are some limitations of the assay. The patients tested were known to have an infected prosthesis and no other known septic foci. If this serological test were to be applied in clinical practice, it would be important to ensure that a positive response was not due to sepsis at other sites. The kinetics of the antibody response have not been studied yet and the difference between the normal early post-operative response and an early infection needs to be clarified. The effect of antibiotic therapy on the serological response also requires study. Crossreactions were observed with sera from patients with other serious infections with gram-positive cocci. Common antigenic determinants residing in cell wall or cell membrane teichoic acids might be responsible. At present the ELISA is not specific for $S$. epidermidis infections. From a clinical point of view this lack of specificity may be an advantage. The most important distinction is between patients with prostheses which are loose as a result of infection and those which are not infected.

This study was supported by a grant from the British Orthopaedic Association Wishbone Trust.

\section{References}

1. Jansen B, Schumacher-Perdreau F, Peters G, Pulverer G. New aspects in the pathogenesis and prevention of polymerassociated foreign body infections caused by coagulase-negative staphylococci. J Invest Surg 1989; 2: 361-380.

2. Ling RSM. Complications of total hip replacement. Edinburgh, Churchill Livingstone. 1984.

3. Krikler SJ, Lambert PA. Extracellular proteins as a potential marker of active Staphylococcus aureus infection in bone. J Med Microbiol 1992; 37: 227-231.

4. Lambert PA, Krikler SJ, Patel R, Parvathan S. Enzyme-linked immunosorbent assay for the detection of antibodies to exocellular proteins of Staphylococcus aureus in bone infection. FEMS Microbiol Lett 1992; 100: 67-70.

5. Krikler SJ, Pennington TH, Petrie D. Typing of strains of Staphylococcus aureus by Western Blot analysis of culture supernates. J Med Microbiol 1986; 21: 169-171.

6. Bell JA, Pennington TH, Petrie DT. Western blot analysis of staphylococcal antibodies present in human sera during health and disease. J Med Microbiol 1987; 23: 95-99.

7. Archer GL. Staphylococcus epidermidis and other coagulasenegative staphylococci. In: Mandell GL, Bennett JE (eds.) Principles of infectious diseases, 3rd edn. New York, Churchill Livingstone. 1990: 1511-1518.

8. Peters G, Locci R, Pulverer G. Adherence and growth of coagulase-negative staphylococci on surfaces of intravenous catheters. J Infect Dis 1982; 146: 479-482.

9. Peters G, Schumacher-Perdreau B, Jansen B, Bey M, Pulverer G. Biology of $S$. epidermidis extracellular slime. Zentralbl Bakteriol Mikrobiol Hyg Abt 1 1987; Suppl 16: 15-32.

10. Tojo M, Yamashita N, Goldmann DA, Pier GB. Isolation and characterization of a capsular polysaccharide adhesin from Staphylococcus epidermidis. J Infect Dis 1988; 157: 713-722.

11. Christensen GD, Barker LP, Mawhinney TP, Baddour LM, Simpson WA. Identification of an antigenic marker of slime production for Staphylococcus epidermidis. Infect Immun 1990; 58: 2906-2911.

12. Mack D, Nedelmann M, Krokotsch A, Schwarzkopf A, Heeseman J, Laufs R. Characterization of transposon mutants of biofilm-producing Staphylococus epidermidis impaired in the accumulative phase of biofilm production: genetic identification of a hexosamine-containing polysaccharide intercellular adhesin. Infect Immun 1994; 62: 3244-3253.

13. Hussain M, Hastings JGM, White PJ. Comparison of cell-wall teichoic acid with high-molecular-weight extracellular slime material from Staphylococcus epidermidis. J Med Microbiol 1992; 37: 368-375.

14. Lambe DW, Ferguson KP, Keplinger JL, Gemmell CG, Kalbfleisch JH. Pathogenicity of Staphylococcus lugdunensis, Staphylococcus schleiferi, and three other coagulase-negative staphylococci in a mouse model and possible virulence factors. Can J Microbiol 1990; 36: 455-463.

15. Jansen B, Schumacher-Perdreau F, Peters G, Pulverer G. Evidence for degradation of synthetic polyurethanes by 
Staphylococcus epidermidis. Int J Med Microbiol 1991; 276: $36-45$.

16. Lugtenberg B, Meijers J, Peters R, van der Hoek P, van Alphen L. Electrophoretic resolution of the 'major outer membrane protein' of Escherichia coli $\mathrm{K} 12$ into four bands. FEBS Lett 1975; 58: 254-258.

17. Sharp J, Poxton IR. Analysis of the membrane lipocarbohydrate antigen of Clostridium difficile by polyacrylamide gel electrophoresis and immunoblotting. FEMS Microbiol Lett 1986; 34: 97-100.

18. Havaei SA, Hancock IC. The capsular turnover product of Staphylococcus aureus strain Smith. FEMS Microbiol Lett 1994; 118: 37-43.

19. Beachey EH, Courtney HS. Bacterial adherence: the attachment of group A streptococci to mucosal surfaces. Rev Infect Dis 1987; 9 suppl 5: 475-481. 